\title{
Urgences
}

\section{À la recherche du temps... intérieur}

Marie Bélisle, Chroniques analogiques, Saint-Lambert, Noroît, 1989, 72 p.

\section{Françoise Daigle}

Numéro 25, octobre 1989

Multiples de Hamlet

URI : https://id.erudit.org/iderudit/025546ar

DOI : https://doi.org/10.7202/025546ar

Aller au sommaire du numéro

Éditeur(s)

Urgences

ISSN

0226-9554 (imprimé)

1927-3924 (numérique)

Découvrir la revue

Citer ce compte rendu

Daigle, F. (1989). Compte rendu de [À la recherche du temps... intérieur / Marie Bélisle, Chroniques analogiques, Saint-Lambert, Noroît, 1989, 72 p.] Urgences,

(25), 81-82. https://doi.org/10.7202/025546ar d'utilisation que vous pouvez consulter en ligne.

https://apropos.erudit.org/fr/usagers/politique-dutilisation/ 


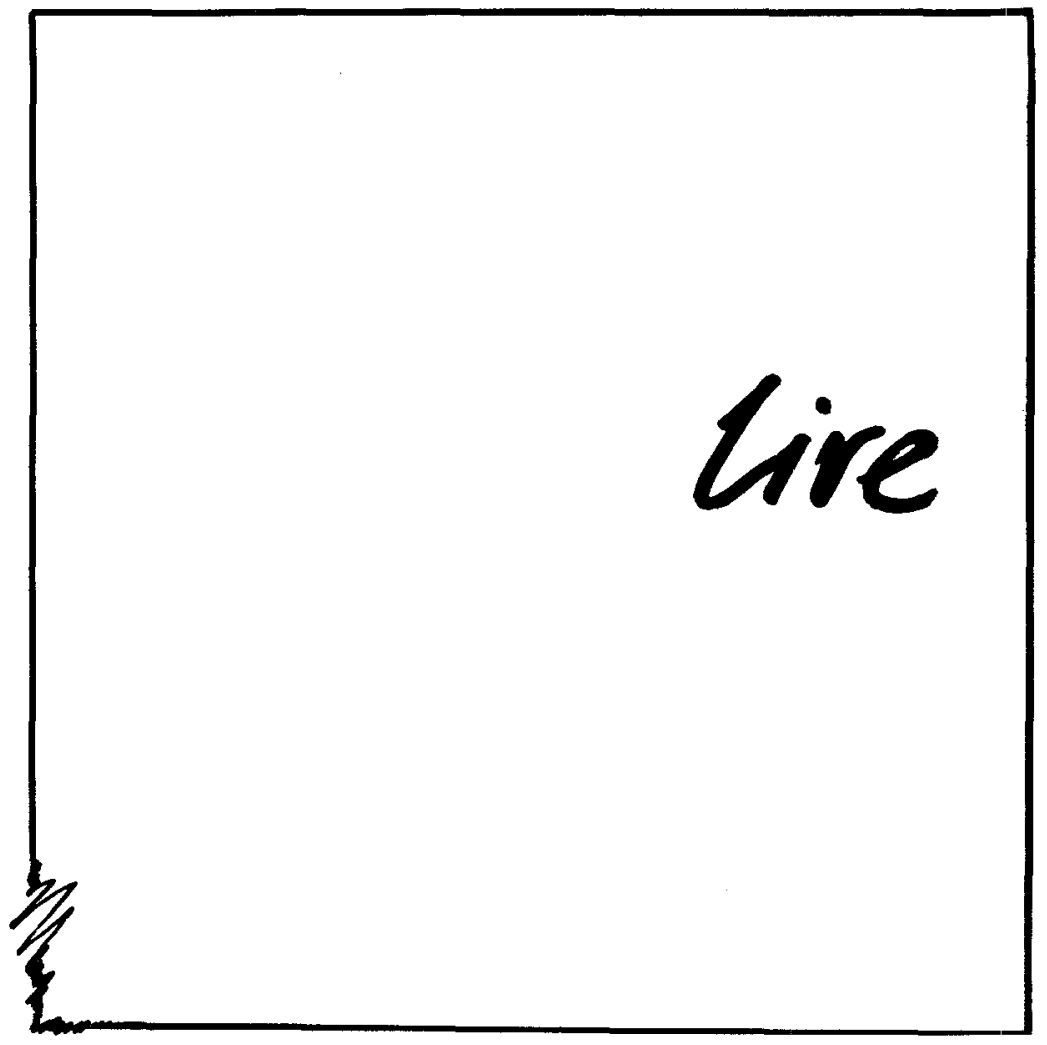




\section{À la recherche du temps... intérieur}

\section{Marie Bélisle, Chroniques analogiques, Saint- Lambert, Noroît, 1989, 72 p.}

Lire - prendre contact avec un nouveau recueil de poèmes, avec un nouvel auteur, demande un apprivoisement semblable au choix d'un nouveau logis. Laisser parler la couleur des tapisseries. Humer l'ambiance. Ne pas se presser. Vérifier si l'intimité habituelle se marie bien à la disposition des pièces. Plus encore que dans un roman - où l'auteur, bavard, nous raconte et nous dit tout - , il faut ici être attentif et savoir lire les images sautillantes sous les lignes.

C'est, quant à moi, une première lecture de Marie Bélisle. Eit pourtant, ni le temps ni l'espace ne nous séparent beaucoup. Il y a ainsi, scuvent, de par l'abondance des cuvres littéraires, des noirs et des blancs crés par le hasard.

Chroniques analogiques est un petit recueil de quelque soixante-douze pages, tout en poèmes courts et rythmés. On nous convie ici, de par le titre, à un récit de "faits historiques, rapportés dans l'ordre de la succession" (dixit Le petit Robert) et, tout à la fois, à un recueil analogique qui s'empressera aussitôt de brouiller la dimension «historique" en y raccrochant les images nées spontanément de la mémoire et de l'imaginaire. Par télescopage implicite, c'est aussi le «chronologique» que l'on se propose d'observer.

Le temps. Le jeu du temps, de l'espace et du désir constitue le pivot du recueil. On s'arrêtera tantôt à vivre la mémoire qui recrée toutes dimensions; tantôt, obstinément, à dénombrer, à compter, à chiffrer l'expérience du temps.

Ainsi, chaque partie nous informe du moment de l'écriture (mais est-ce bien de cela qu'il s'agit). La première partie du recueil, intitulée «Le jeu du dénombrement", s'amuse à recenser en douze poèmes les douze mois de l'année. On ne peut être plus sage ni plus respectueux de l'ordre; les quatrains bien numérotés nous proposent logiquement de glisser de janvier à décembre. Mais toutefois, dans ce déroulement (dénombrement), les lettres dansent déjà en allitérations, homophonies et anagrammes. Et si le jeu se veut mathématique et en emprunte même le langage, c'est pour en démontrer aussi l'ineptie: pour "déplacer l'axe des éphémérides» (p. 11), «créer la fièvre divisible par quatre» (p. 12), «joindre les signes négatifs qui/doubles s'annulent» (p. 16). Et encore, à chaque poème, la perversion de l'ordre par la fièvre, le désir, *le flux du sang premièrement» (p. 11).

La seconde partie, "Lexies quantièmes", annonce clairement ses couleurs (p. 25):

de quoi s'agit-il

transgresser la loi métrique anachronique morte

déconstruire le lieu codé siècle par siècle

relier relire les semaines mot par mot

mois par mois désir année

Dans ces douze nouveaux poèmes, c'est le corps - l'objet du désir - qui prend doucement sa place. Le temps s'immisce encore au hasard de la couleur des mots, mais c'est l'amour, l'oubli, la fusion qui en font la trame. Ainsi l'on ose uobstinément croire à la pertinence du plaisir» (p. 33), cette négation de 
la chronologie. Et l'on se laisse emporter par la passion, pour, comme à regret, "calmement préparer la fermeture de l'an*, pour se soumettre à l'inexorable, mais en signant au passage le triomphe de l'analogie, cette «odeur obscure " qui « régit antérieure / sur la peau » (p. 36). À mi-chemin de ce recueil, déjà les jeux sont faits: parti pris pour «le juste retour des choses *, l'évocation, la mémoire infinie.

C'est dans l'«anachronique * que l'on s'engage désormais, dans le langage du corps, de la tendresse, afin de «traduire l'osmose» (p. 47). «Ça passe par le sommeil tout bas / par l'histoire que l'on raconte aux petites" (p. 42), nous dit-on dans de très beaux vers. Les lignes droites et mathématiques sont devenues «courbes * et * parcours optique * (p. 39); et les poèmes sont présentés sous forme d'un semainier déroutant, qui revient en arrière, reprend, s'embrouille savamment. Petite mécanique appliquée à revanche sur la date - le jour, l'heure, la seconde; semaine ébréchee qui se fissure de partout.

Les «imprécis décalages» voyagent entre Paris et Montréal, par «imprécises allongées * et «imprécises expresses * comme le café (expresso/allonge), nous explique-t-on (p. 64). Ici, les «imprécises allongées » nous livrent d'emblée toutes les clés du jeu; s'arrêtent cette fois à la notion du temps/ espace, de la distance.

«Le son le papier sur la peau (p. 56) recréent le réseau du temps intérieur, l'authenticité de «ses petites alliances" (p. 59) éminemment significatives. Jouent du télescopage, du découpage, du collage; trichent ingenieusement de toutes les façons possibles. Et me séduisent infiniment, peut-être par la générosité de l'auteure qui ne perd rien à nous démontrer $s a$ manière de comprendre le temps; qui applique un système plus fou et plus vivant que le si terne calendrier.

Que reste-t-il à sacrifier encore sinon l'absurdité de la comptabilité chronologique, sa futilité lorsqu'elle est confrontée au mouvement du désir... Dans cette « Seconde avancée $"$, cinquième partie qui abandonne pour de bon la symétrie, cette incompétence temporelle éclate avec ironie. Une histoire, en quelques poèmes, de séduction (séduction descriptive, vue du dehors, froide et... calculée). En tête de chaque poème, comme une didascalie, une note de script: «(3 sec.)» (p. 67), «(4 sec.)» (p. 68), et ainsi de suite. Comme des séquences filmées. Et toute l'incongruité de cette notation plaquée sur le mouvement des corps (les indications ne correspondent d'ailleurs absolument ni au temps «réel», ni à la vitesse de lecture). C'est finalement la chute du recueil, sur la jouissance consommée. Petite mort non agréée dans le cadre rigide de nos existences où l'on ne meurt qu'une fois.

\section{Et l'on peut recommencer ainsi perpétuellement...}

Chroniques analogiques est un recueil aéré et accueillant, tant par l'espace blanc laissé à chaque page pour nos silences et nos méditations que par les dessins de l'auteure, aussi éloquents (que l'on peut lire aussi bien) que les poèmes. Limpression que chaque mot trouve la place qui lui revient. Rien de lourd, rien qui accroche, rien qui « dépasse».

Somme toute, un logement clair où la lumière circule bien, que l'on habitera aisément en s'y ménageant des coins plus ombragés pour l'amour, en repérant les endroits stratégiques où suspendre ses graffitis personnels. 